\title{
Integration of Biological, Psychological, and Social Aspects in Agent-Based Simulation of a Violent Psychopath
}

\author{
Tibor Bosse, Charlotte Gerritsen, and Jan Treur \\ Vrije Universiteit Amsterdam, Department of Artificial Intelligence \\ De Boelelaan 1081a, NL-1081 HV, Amsterdam, The Netherlands \\ \{tbosse, cg, treur\}@few.vu.nl \\ http://www.few.vu.nl/ \{tbosse, cg, treur\}
}

\begin{abstract}
In the analysis of criminal behaviour, a combination of biological, psychological and social aspects may be taken into account. Dynamical modelling methods developed in recent years often address these aspects separately. This paper contributes an agent-based modelling approach for behaviour of a certain criminal type, the violent psychopath, in which these aspects are integrated in one dynamical system. It is shown how within a certain social context, an interaction between biological factors and cognitive and emotional factors can lead to a crime committed when an opportunity is perceived.
\end{abstract}

Keywords: simulation, violent behaviour, BDI-models, integration of biological, psychological and social aspects.

\section{Introduction}

Analysis of criminal behaviour is a central issue in Criminology. Such an analysis involves different types of aspects, such as biological, psychological, and social aspects, and their mutual interactions; e.g., [2, 5, 8, 10, 12]. Usually such analyses are made by criminologist researchers or practitioners in a nonexact manner, without using any formalisation or computer support.

In recent years, much progress has been made in biological, cognitive, and social complex dynamical systems modelling within areas such as Artificial Intelligence, Computational Biology and Computational Cognitive Science. The methods developed in these areas usually address one of the disciplines separately. However, when an integrated modelling approach is applied, this opens the perspective to address the analysis of criminal behaviour in more exact, formalised and computer supported manners. Thus, the way is paved to a more solid basis and computer support for simulation and analysis in the area of Criminology. The research discussed here explores this potential. It identifies on the one hand useful knowledge from the literature in Criminology and the different disciplines underlying it e.g., $[6,8,10]$, and on the other hand it exploits dedicated agent-based and dynamical systems modelling techniques [3]. The aim is, by combining these, to develop an 
integrated computer-supported method for the analysis of certain criminal behaviour types, in this case the violent psychopath, as described in the literature.

To model all of the aspects mentioned above in an integrated manner, both qualitative aspects (e.g., beliefs, desires, intentions, certain brain deviations, and the presence of certain agents), and quantitative aspects (e.g., hormone levels, distances and time durations) have to be addressed. The modelling language LEADSTO [3] fulfils these desiderata. In LEADSTO, basic (atomic) state properties can have a qualitative, logical format, such as an expression desire(d1), expressing that desire d1 occurs, or a quantitative, numerical format such as an expression has_value $(x, v)$ which expresses that variable $\mathrm{x}$ has value $\mathrm{v}$. Such atomic state properties can be combined to more complex state properties by taking conjunctions by means of the logical operator 'and'. Based on these state properties, dynamic properties can be expressed as follows. Let $\alpha$ and $\beta$ be state properties of the form 'conjunction of ground atoms or negations of ground atoms'. In the LEADSTO language the notation $\alpha \rightarrow_{\mathrm{e}, \mathrm{f}, \mathrm{g}, \mathrm{h}} \beta$, means:
If
state property $\alpha$ holds for a certain time interval with duration $g$,
then after some delay (between e and $f$ ) state property $\beta$ will hold for a certain time interval of length $h$.

In this paper, Section 2 discusses a specific type of criminal used as a case study: the violent psychopath. In Section 3 the simulation model based on LEADSTO is presented. Section 4 discusses some of the simulation results for the case study. Finally, Section 5 is a discussion about the approach and its possible applications.

\section{Case Study: Violent Psychopath}

The case study made in this paper focuses on a specific kind of violent offender: the violent psychopath, e.g., [2, pp. 87-120; 8, pp. 123-183; 9; 10; 12, pp. 193-207]. In this section, this type of criminal is briefly introduced, by subsequently addressing psychological, social and biological aspects.

Psychological Aspects. Psychopaths do not show feelings like the rest of us. They lack the normal mechanisms of anxiety arousal, which ring alarm bells of fear in most people. Confronted with trial and danger, even their skin does not sweat and become clammy like the skin of normal people [8, p.157]. Violent psychopaths, who are almost always males, can be described as predators and are usually proud of it. Their kind of violence is similar to predatory aggression, that is accompanied by minimal or no sympathetic arousal and is planned, purposeful, and without emotion. This is correlated with a sense of superiority; they like to exert power and have unrestricted dominance over others, ignoring their needs and justifying the use of whatever they feel compelling to achieve their goals and avoid adverse consequences for their acts. An important trigger for psychopathic violent behaviour is the use of drugs and/or alcoholism. They are more likely to turn to drink and drugs and their brain reacts in a different way to the effects of drugs and alcohol. For a psychopath, using drugs or alcohol can become a compulsion and, through a genetic and neurological mechanism, result in violent behaviour [8, p.201].

Social Aspects. Psychopaths are characterised by a disregard for social obligation and a lack of concern for the feelings of others. They display pathological egocentricity, 
shallow emotions, lack of insight and remorse, anxiety or guilt in relation to their antisocial behaviour. They are usually callous, manipulative individuals, incapable of lasting friendship and of love. They use charm, manipulation, intimidation and violence to control others and to satisfy their own selfish needs. Lacking in conscience and in feelings for others, they violate social norms and expectations without the slightest sense of guilt or regret.

Biological Aspects. Psychopaths have a specific deviation in the brain: the frontal lobes are disconnected from the limbic area. The frontal lobes are the area of the brain that is concerned with conscience, guilt and remorse and is the residence of our morality. The limbic area generates feelings [8, p.157]. Because of the disconnection, psychopaths cannot express their emotions in terms of feeling. They know the difference between right and wrong, but the difference does not matter for them. It is hard for a psychopath to understand or imagine the pain of other people [8, p.158]. Furthermore, violent psychopaths have a high level of testosterone, which makes them more aggressive in their behaviour, and low levels of serotonin, which makes them easily bored and impulsive, and stimulates them to seek sensation. Once they reach adulthood, their condition is incurable. However, only a fraction of psychopaths develops into violent criminals [8, p. 267].

\section{The Integrated Simulation Model}

In this section, the integrated simulation model that has been developed is described in more detail. The agent-based model has been built by composing four submodels for different aspects. The first submodel is a model that bases the preparation and performing of actions on beliefs, desires and intentions (BDI); e.g., [11]. Some of the relations within this BDI-model (as depicted by arrows in graphical format in Figure 1) can be specified in formal LEADSTO format as follows:

desire(d1) $\wedge$ belief(satisfies(a1, d1)) $\rightarrow 0.2,0.2,1,1$ intention(a1) intention(a1) ^ belief(opportunity_exists_for(a1)) $\rightarrow_{0.2,0.2,1,1}$ to_be_performed(a1)

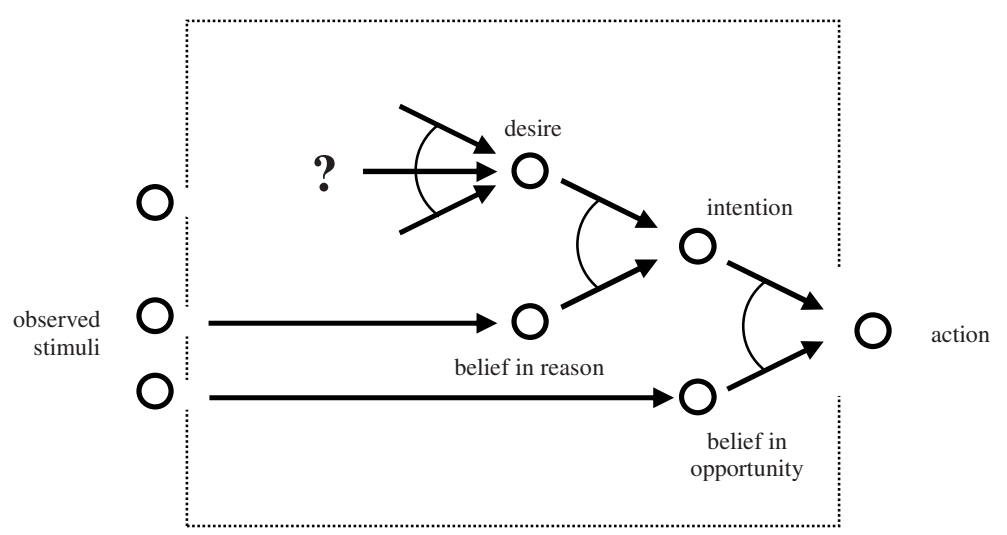

Fig. 1. Structure of the Generic BDI-model 
Note that the beliefs used here both depend on observed stimuli, as shown in Figure 1. Furthermore, $\wedge$ stands for the conjunction operator (and) between the atomic state properties (depicted below by an arc connecting two (or more) arrows).

A second submodel is used to determine desires, needed as input for the BDImodel. This submodel incorporates various biological and psychological aspects and their interactions. The biological and psychological aspects involved are of different types. On the one hand there are qualitative aspects, such as anatomical aspects concerning brain deviations (e.g., the absence of certain connections). On the other hand there are quantitative aspects, such as biochemical aspects concerning testosterone levels. To model these, both logical relations (as in qualitative modelling) and numerical relations (as in differential equations) have been integrated in one modelling framework.

A third submodel determines how observations lead to beliefs in an opportunity as needed as input for the BDI-model. The notion of opportunity is based on criteria as indicated in the Routine Activity Theory by [5]: a suitable target and the absence of a suitable guardian. This was specified by the following dynamic property in LEADSTO format:

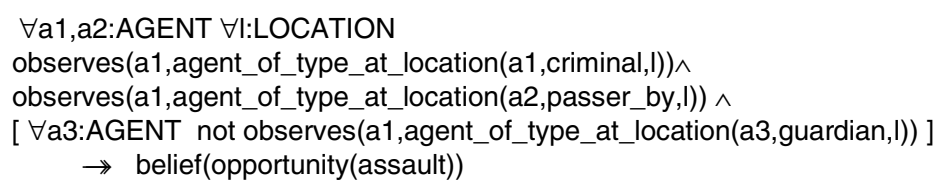

A fourth submodel is a physical and social environment model of the world involving an environment (modelled here as a graph with 8 nodes, called location A though $\mathrm{H}$ ) in which a number of agents move around and sometimes meet at the same location. One of the agents represents the criminal type that is considered (violent psychopath), the other agents represent potential victims (passers-by) and guardians. At some locations a suitable target can be found, for example an agent that looks rich and/or weak. However, as also the guardians are moving around, such targets may be protected, whenever at the same location a guardian is observed by the criminal. This models the aspect of social control. To model the dynamics of the agents moving around in the environment, a number of dynamic properties are used that relate successive states to each other.

Note that the first three submodels describe the internal processes of the agent representing the criminal type considered: the violent psychopath. The other submodel describes the physical and social environment.

\section{An Example Simulation Trace}

A number of simulation traces have been generated for the behaviour of the agent representing the considered violent psychopath criminal type under different circumstances. In this section, one specific simulation trace is described in detail. For this trace, the following initial state properties have been chosen for the violent psychopath agent: the testosterone level during pregnancy is high, the basic adrenalin level is medium (value 5), the basic level of serotonin is low (value 3), the basic level of oxytocine is low, and the person's thymus gland is not developed properly. 
Moreover, the brain is configured for the following characteristics: a sensitivity for alcohol, a high anxiety threshold (value 8), a high excitement threshold (value 8), a low positive emotional attitude towards others and a low negative emotional attitude towards others. In addition, some inputs for the model over time are provided: initially there is a rather neutral stimulus present, which is not very dangerous nor exciting (both aspects have value 2), alcohol is used during the whole scenario, no Prozac is taken and no Ritalin is taken. Finally, the initial characteristics of the environment are: the violent psychopath agent is at location $\mathrm{A}$, there is a guardian at location $\mathrm{C}$, and one at location $\mathrm{E}$ and there is a passer-by at location $\mathrm{F}$, and one at location $\mathrm{G}$.

In Figure 2, the results of the chosen example simulation trace (run) for the behaviour of the violent psychopath agent are shown. In these pictures, time is on the horizontal axis; the state properties are on the vertical axis. A dark box on top of the line indicates that the property is true during that time period, and a lighter box below the line indicates that the property is false. Figure 2 depicts the biological/psychological aspects of the behaviour of the violent psychopath agent within the example simulation trace, such as change of serotonin levels, and the generation of beliefs, desires and intentions. Due to space limitations the environmental aspects are not shown in Figure 2.

As can be seen in Figure 2, the initial settings mentioned above lead to the following characteristics in the psychopath criminal type agent: the insulin level is high, the anxiety threshold is high (value 8), the excitement threshold is high (value 10), the person is sensitive for alcohol, and his emotional attitude towards others, both positive and negative, is low. In addition, he has a low neural self. This leads to a low me-other differentiation, a low empathy, and eventually, a low theory of mind. His high initial level of testosterone leads to a high current level of testosterone, which in turn leads to high aggressiveness. Moreover, the medium level of initial adrenalin leads to a medium current level of adrenalin (value 5). This current level of adrenalin, combined with a low level of oxytocine, leads to a low desire to cope anxiety and a low desire to ignore anxiety. Furthermore, the low initial serotonin level (value 3) leads to a low current level of serotonin (also value 3 ). This current level of serotonin combined with sensitivity for alcohol and taking alcohol leads (at time point 2) to a decreased level of serotonin (value 0). The current serotonin level also leads to an increased excitement threshold (from value 10 to value 13). The high insulin level leads to a low blood sugar level and a high impulsiveness.

When the excitement threshold is higher than the strength of the observed stimuli, then the violent psychopath agent will become bored. This leads to the desire for actions with strong stimuli at time point 4 . As a result of this desire and several other characteristics mentioned above, the agent eventually (time point 5) develops a desire for an action that is characterised by the following aspects: a low theory of mind, high aggressiveness, a low desire to cope with anxiety, a low desire to ignore anxiety, a high desire for actions with strong stimuli, high impulsiveness, a low positive attitude towards others and a low negative attitude towards others. This desire, combined with the belief that assaulting someone will lead to the satisfaction of such a desire, leads to the intention to assault someone at time point 6 . 


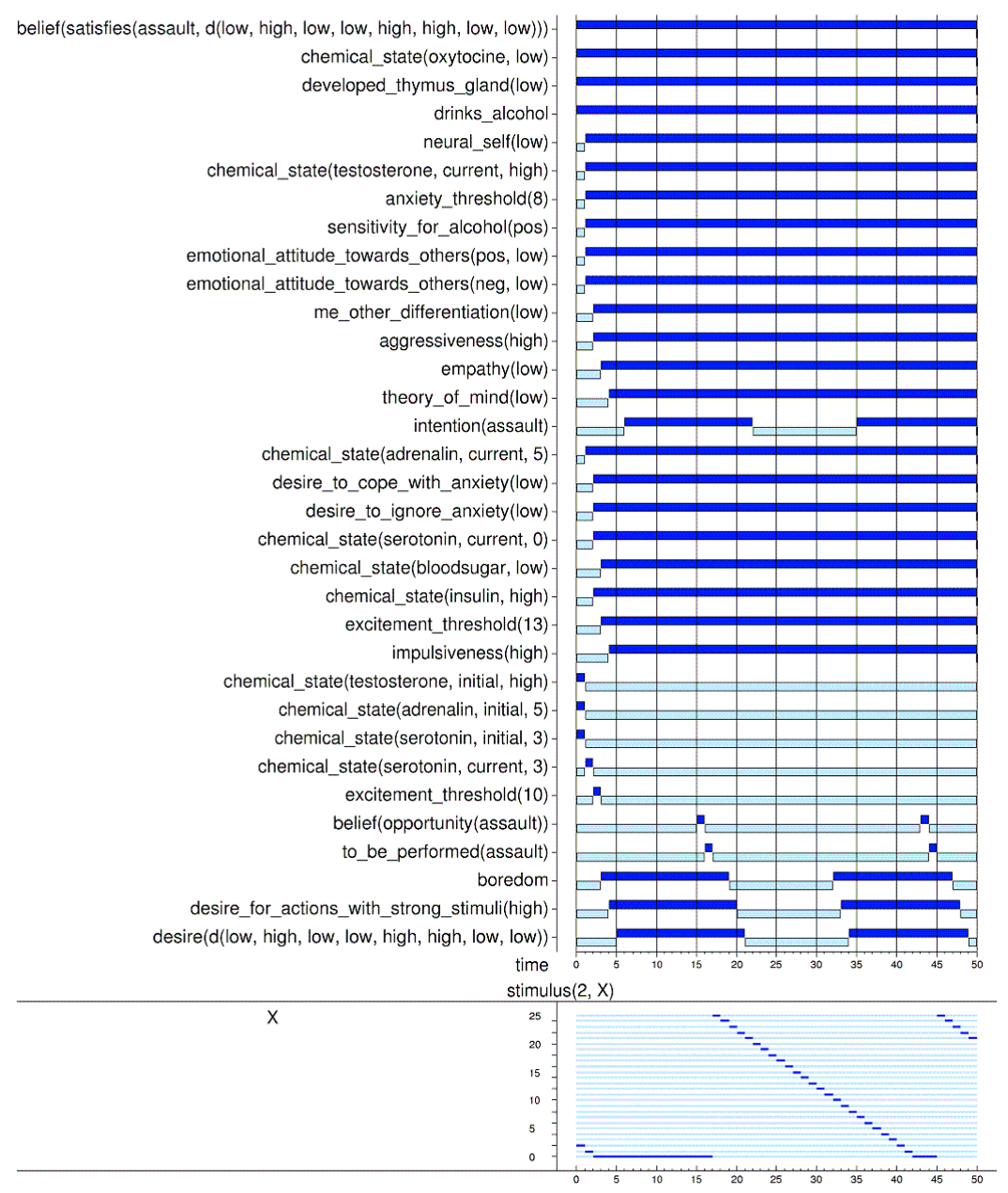

Fig. 2. The example simulation trace: biological and psychological aspects

In the meantime, the criminal agent has started to move around in the world. In total, in this example trace, which was kept simple for reasons of presentation, there are 5 agents in the world: agent 1 representing the considered criminal type (i.e., the violent psychopath agent described in Figure 2, agents 2 and 3 are guardians, and agents 4 and 5 are passers-by (i.e., potential victims). These agents are moving through the world. For example, agent 1 starts at location A (time point 0), then moves to location B (time point 4), and so on. When the criminal agent meets a passer-by without a guardian present then the criminal agent will believe that there is an opportunity to assault the passer-by. There is an opportunity to assault a passer-by at time point 15 . This opportunity has arisen because agent 1 is at location $G$ and agent 5 is also at this location, and there are no guardians present (agents 2 and 3 are respectively at location $\mathrm{E}$ and $\mathrm{H}$ ). At time point 43 there is another opportunity for 
agent 1 to assault someone. This is because agent 1 is at location $\mathrm{H}$ together with agent 5 , and agents 2 and 3 are not present.

In Figure 2, the psychopath agent's beliefs about opportunities are also depicted. When such a belief is present, together with the intention to assault someone, the actual action to assault the passer-by is performed. This happens twice in the trace: at time point 16 and 44. Finally, note that, when a violent psychopath agent assaults someone, this significantly raises the level of stimuli he experiences. The values of these stimuli are shown in the bottom part of Figure 2. When this value passes his excitement threshold, he will stop being bored. As a consequence, also his desire for actions with strong stimuli will be fulfilled, and his desire and intention for an action that is characterised by this desire (among others) will disappear. However, after a while, the increased value of the experienced stimuli will gradually decrease and the psychopath agent will be bored again. This will lead to new desires, new intentions, and eventually (at time point 44), to a new assault.

\section{Discussion}

This paper proposes a method to analyse the behaviour of certain criminal types as described in the literature, based on integrated dynamic modelling. As a case study, this method has been applied to analyse the behaviour of the violent psychopath criminal type. It has been found that the model indeed shows the behaviour as described for this criminal type. The model takes into account a cognitive modelling approach to the preparation of actions based on beliefs, desires and intentions (BDI) in a more or less standard manner e.g., [11]. However, for this standard BDI-model, desires and beliefs about opportunities are required as input. Concerning the former, additional biological, cognitive, and emotional aspects have been used as a basis to generate desires. For the latter, additional social aspects have been used to generate beliefs on opportunities based on two specific criteria (suitable target, presence of guardian) as indicated by the Routine Activity Theory in [5]. For the generation of desires various other aspects as described in the literature are taken into account, varying from specific types of brain deviations, and serotonin and testosterone levels, to the extent to which me-other differentiation and a theory of mind were developed. Thus the model integrates biological, cognitive and socially related aspects in the process of desire generation, as extracted from literature, in particular [6, 8, 10]. These involve both qualitative aspects (such as the anatomy of brain deviations, and presence or absence of agents at a specific location in the world), and quantitative aspects (such as distances and time durations in the world and hormone ands neurotransmitter levels).

To achieve the integration of different aspects, the proposed modelling approach (based on the LEADSTO language) integrates qualitative, logical aspects and quantitative, numerical aspects. This integration allows one to exploit techniques from both areas. As the latter type of aspects are fully integrated in the former, this results in a declarative specification for which automated methods for logical analysis can be exploited. Conversely, as the former types of aspects are fully integrated in the latter, a simulation environment is offered that extends the usual possibilities to simulate dynamical systems using numerical methods, by incorporating qualitative elements. 
Only few papers on simulation of criminal behaviour can be found in the literature, and they usually address a more limited number of aspects than the modelling approach presented in this paper. For example, Brantingham and Brantingham, [4] discuss the possible use of agent modelling approaches to criminal behaviour in general, but do not report a specific model or case study. Moreover, in [1] a model is presented with emphasis on the social network and the perceived sanctions. However, this model leaves the psychological and biological aspects largely unaddressed. The same applies to the work reported in [7], where the emphasis is on the environment, and police organisation.

\section{References}

1. Baal, P.H.M. van (2004). Computer Simulations of Criminal Deterence: from Public Policy to Local Interaction to Individual Behaviour. Ph.D. Thesis, Erasmus University Rotterdam. Boom Juridische Uitgevers.

2. Bartol, C.R. (2002). Criminal Behavior: a Psychosocial Approach. Sixth edition. Prentice Hall, New Jersey.

3. Bosse, T., Jonker, C.M., Meij, L. van der, and Treur, J. (2005). LEADSTO: a Language and Environment for Analysis of Dynamics by SimulaTiOn. In: Eymann, T. et al. (eds.), Proc. of the $3^{\text {rd }}$ German Conference on Multi-Agent System Technologies, MATES'05. LNAI 3550. Springer Verlag, 2005, pp. 165-178. Extended version to appear in International Journal of Artificial Intelligence Tools, 2007.

4. Brantingham, P. L., \& Brantingham, P. J. (2004). Computer Simulation as a Tool for Environmental Criminologists. Security Journal, 17(1), 21-30.

5. Cohen, L.E. and Felson, M. (1979). Social change and crime rate trends: a routine activity approach. American Sociological Review, vol. 44, pp. 588-608.

6. Delfos, M.F. (2004). Children and Behavioural Problems: Anxiety, Aggression, Depression and ADHD; A Biopsychological Model with Guidelines for Diagnostics and Treatment. Harcourt book publishers, Amsterdam.

7. Melo, A., Belchior, M., and Furtado, V. (2005). Analyzing Police Patrol Routes by Simulating the Physical Reorganisation of Agents. In: Sichman, J.S., and Antunes, L. (eds.), Multi-Agent-Based Simulation VI, Proceedings of the Sixth International Workshop on Multi-Agent-Based Simulation, MABS'05. Lecture Notes in Artificial Intelligence, vol. 3891, Springer Verlag, 2006, pp 99-114.

8. Moir, A., and Jessel, D. (1995). A Mind to Crime: the controversial link between the mind and criminal behaviour. London: Michael Joseph Ltd; Penguin.

9. Quay, H.C. (1965). Psychopathic Personality: Pathological Stimulation-Seeking. American Journal of Psychiatry, vol. 122, pp. 180-183.

10. Raine, A. (1993). The Psychopathology of Crime: Criminal Behaviors as a Clinical Disorder. New York, NY: Guilford Publications.

11. Rao, A.S. \& Georgeff, M.P. (1991). Modelling Rational Agents within a BDI-architecture. In: Allen, J., Fikes, R. and Sandewall, E. (eds.), Proceedings of the Second International Conference on Principles of Knowledge Representation and Reasoning, (KR'91). Morgan Kaufmann, pp. 473-484.

12. Turvey, B. (1999). Criminal Profiling: an Introduction to Behavioral Evidence Analysis. Academic Press. 